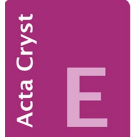
COMMUNICATIONS

ISSN 2056-9890

\section{Crystal structure of ethyl $1^{\prime}, 1^{\prime \prime}$-dimethyl- 2",3-dioxo-3H-dispiro[benzo[b]thio- phene-2,3'-pyrrolidine-2', $3^{\prime \prime}$-indoline]-4'- carboxylate}

\author{
M. P. Savithri, ${ }^{a}$ M. Suresh, ${ }^{b}$ R. Raghunathan, ${ }^{b}$ R. Raja ${ }^{c}$ and \\ A. SubbiahPandi ${ }^{\mathrm{C}}$ * \\ adDepartment of Physics, Queen Mary's College (Autonomous), Chennai 600004 , \\ India, 'bepartment of Organic Chemistry, University of Madras, Guindy Campus, \\ Chennai 600 025, India, and ${ }^{\mathbf{C}}$ Department of Physics, Presidency College \\ (Autonomous), Chennai 600005 , India. ${ }^{*}$ Correspondence e-mail: \\ aspandian59@gmail.com
}

Received 16 January 2015; accepted 30 January 2015

Edited by H. Stoeckli-Evans, University of Neuchâtel, Switzerland

In the title compound, $\mathrm{C}_{23} \mathrm{H}_{22} \mathrm{~N}_{2} \mathrm{O}_{4} \mathrm{~S}$, the pyrrolidine ring has an envelope conformation with the spiro $\mathrm{C}$ atom, shared with the indoline ring system, as the flap. The mean planes of the benzothiophene and indoline ring systems are inclined to the mean plane of the pyrrolidine ring by 88.81 (8) and $79.48(8)^{\circ}$, respectively, and to each other by $68.12(5)^{\circ}$. In the crystal, molecules are linked via $\mathrm{C}-\mathrm{H} \cdots \mathrm{O}$ hydrogen bonds, forming chains propagating along [001].

Keywords: crystal structure; dispiro; benzothiophene; pyrrolidine; indole; $\mathrm{C}-\mathrm{H}$... O hydrogen bonds.

CCDC reference: 1046459

\section{Related literature}

For various biological activities of indole derivatives, see: Harris \& Uhle (1960); Ho et al. (1986); Stevenson et al. (2000). For the crystal structures of two very similar compounds, see: Savithri et al. (2014).

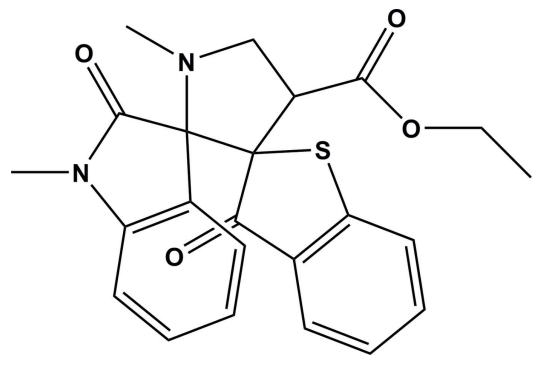

\section{Experimental}

2.1. Crystal data

$\mathrm{C}_{23} \mathrm{H}_{22} \mathrm{~N}_{2} \mathrm{O}_{4} \mathrm{~S}$

$M_{r}=422.49$

Monoclinic, $C 2 / c$

$a=23.7049$ (11)

$b=8.2632$ (3) A

$c=22.1003(8) \AA$

$\beta=102.337(2)^{\circ}$

$V=4229.0(3) \AA^{3}$

$Z=8$

Mo $K \alpha$ radiation

$\mu=0.19 \mathrm{~mm}^{-1}$

$T=293 \mathrm{~K}$

$0.35 \times 0.30 \times 0.30 \mathrm{~mm}$

2.2. Data collection

Bruker Kappa APEXII CCD diffractometer

Absorption correction: multi-scan (SADABS; Bruker, 2004)

$T_{\min }=0.896, T_{\max }=0.910$ 4621 independent reflections 3869 reflections with $I>2 \sigma(I)$ $R_{\text {int }}=0.029$

\subsection{Refinement}

$R\left[F^{2}>2 \sigma\left(F^{2}\right)\right]=0.038$

$w R\left(F^{2}\right)=0.105$

$S=1.03$

4618 reflections

275 parameters

$\mathrm{H}$-atom parameters constrained

$\Delta \rho_{\max }=0.40 \mathrm{e} \AA^{-3}$

$\Delta \rho_{\min }=-0.20{\mathrm{e} \AA^{-3}}^{-3}$

Table 1

Hydrogen-bond geometry $\left(\AA{ }^{\circ}\right)$.

\begin{tabular}{lllll}
\hline$D-\mathrm{H} \cdots A$ & $D-\mathrm{H}$ & $\mathrm{H} \cdots A$ & $D \cdots A$ & $D-\mathrm{H} \cdots A$ \\
\hline $\mathrm{C} 5-\mathrm{H} 5 \cdots \mathrm{O}^{\mathrm{i}}$ & 0.93 & 2.46 & $3.212(2)$ & 138 \\
\hline
\end{tabular}

Symmetry code: (i) $x,-y, z-\frac{1}{2}$.

Data collection: $A P E X 2$ (Bruker, 2004); cell refinement: $A P E X 2$ and SAINT (Bruker, 2004); data reduction: SAINT and XPREP (Bruker, 2004); $\operatorname{program}(\mathrm{s})$ used to solve structure: SHELXS97 (Sheldrick, 2008); program(s) used to refine structure: SHELXL97 (Sheldrick, 2008, 2015); molecular graphics: ORTEP-3 for Windows (Farrugia, 2012); software used to prepare material for publication: SHELXL97 and PLATON (Spek, 2009).

\title{
Acknowledgements
}

MPS and ASP thank Dr Babu Vargheese, SAIF, IIT, Madras, India, for his help with the data collection.

Supporting information for this paper is available from the IUCr electronic archives (Reference: SU5065).

\section{References}

Bruker (2004). APEX2, SAINT, XPREP and SADABS. Bruker AXS Inc, Madison, Wisconsin, USA.

Farrugia, L. J. (2012). J. Appl. Cryst. 45, 849-854.

Harris, L. S. \& Uhle, F. C. (1960). J. Pharmacol. Exp. Ther. 128, 353-363.

Ho, C. Y., Haegman, W. E. \& Perisco, F. (1986). J. Med. Chem. 29, 118-121.

Savithri, M. P., Suresh, M., Raghunathan, R., Vimala, G., Raja, R. \& SubbiahPandi, A. (2014). Acta Cryst. E70, 94-97.

Sheldrick, G. M. (2008). Acta Cryst. A64, 112-122.

Sheldrick, G. M. (2015). Acta Cryst. C71, 3-8.

Spek, A. L. (2009). Acta Cryst. D65, 148-155.

Stevenson, G. I., Smith, A. L., Lewis, S., Michie, S. G., Neduvelil, J. G., Patel, S., Marwood, R., Patel, S. \& Castro, J. L. (2000). Bioorg. Med. Chem. Lett. 10, 2697-2699. 


\title{
supporting information
}

Acta Cryst. (2015). E71, o142 [doi:10.1107/S2056989015002042]

\section{Crystal structure of ethyl $1^{\prime}, 1^{\prime \prime}$-dimethyl-2",3-dioxo-3H-dispiro[benzo[b]thio- phene-2,3'-pyrrolidine-2',3'-indoline]-4'-carboxylate}

\author{
M. P. Savithri, M. Suresh, R. Raghunathan, R. Raja and A. SubbiahPandi
}

\section{S1. Comment}

Indole compounds can be used as bioactive drugs (Stevenson et al., 2000). Indole derivatives exhibit antiallergic, central nervous system depressant and muscle relaxant properties (Harris \& Uhle, 1960; Ho et al., 1986). In view of this biological importance, the crystal structure of the title compound was determined and the results are presented here.

The X-ray analysis confirms the molecular structure and atom connectivity as illustrated in Fig. 1. The geometric parameters of the title molecule agrees well with those reported for similar structures (Savithri et al., 2014).

The five membered ring $(\mathrm{N} 1 / \mathrm{C} 1 / \mathrm{C} 6-\mathrm{C} 8)$ in the indole moiety adopts an envelope conformation with the $\mathrm{C} 8$ as the flap atom [puckering parameters $\mathrm{q}_{2}=0.0888(2) \AA$ and $\varphi_{2}=284.2(1)^{\circ}$ ] and the pyrrolidine ring (N2/C8-C11) exhibits a twisted conformation [puckering parameters, $\mathrm{q}_{2}=0.4626(2) \AA$ and $\varphi_{2}=233.4(2)^{\circ}$ ]. The bond length $\mathrm{C} 12-\mathrm{O} 2=1.211 \AA$ indicates a keto group in the benzothiophene. The sum of angles at $\mathrm{N} 2$ of the pyrrolidine ring $\left(339^{\circ}\right)$ is in accordance with $s p^{3}$ hybridization and the sum of angles at $\mathrm{N} 1$ of the indole moiety $\left(359^{\circ}\right)$ is in accordance with $s p^{2}$ hybridization.

The pyrrolidine ring (N2/C8-C11) is perpendicular with benzothiophene (C11-C18/S1) oriented at a dihedral angle of $88.81(8)^{\circ}$ and is oriented with indole ring (N1/C1-C8) at a dihedral angle of $79.48(8)^{\circ}$. The thiophene ring (C11$\mathrm{C} 14 / \mathrm{S} 1)$ makes dihedral angles of $87.98(8)^{\circ}$ and $67.14(6)^{\circ}$ with pyrrolidine (N2/C8-C11) and indole (N1/C1-C8) rings, respectively.

In the crystal, hydrogen-bonded chains running along [001] are generated by connecting neighbouring molecules via C$\mathrm{H} \cdots \mathrm{O}$ hydrogen bonds (Table 1 and Fig. 2).

\section{S2. Experimental}

(E)-ethyl 2-(3-oxobenzo[b]thiophen-2(3H)-ylidene) acetate $(1.0 \mathrm{mmol}), \mathrm{N}$-methyl isatin $(1.1 \mathrm{mmol})$ and sarcosine $(1.1$ $\mathrm{mmol})$ were refluxed in methanol $(20 \mathrm{ml})$ until completion of the reaction monitored by TLC analysis. After completion of the reaction the solvent was evaporated under reduced pressure. The crude reaction mixture was dissolved in dichloromethane $(2 \times 50 \mathrm{ml})$ and washed with water followed by brine solution. The organic layer was separated and dried over sodium sulfate. After filtration the organic solvent was evaporated under reduced pressure. The product was separated by column chromatography using hexane and ethyl acetate (9:1) as an eluent to give a colourless solid. The product was dissolved in chloroform $(3 \mathrm{ml})$ and heated for $2 \mathrm{~min}$. The resulting solution was subjected to crystallization by slow evaporation of the solvent giving in single crystals suitable for X-ray crystallographic studies.

\section{S3. Refinement}

All $\mathrm{H}$ atoms were fixed geometrically and allowed to ride on their parent $\mathrm{C}$ atom: $\mathrm{C}-\mathrm{H}=0.93-0.98 \AA$ with $U_{\text {iso }}(\mathrm{H})=$ $1.5 U_{\mathrm{eq}}(\mathrm{C})$ for methyl $\mathrm{H}$ atoms and $=1.2 U_{\mathrm{eq}}(\mathrm{C})$ for other $\mathrm{H}$ atoms. 


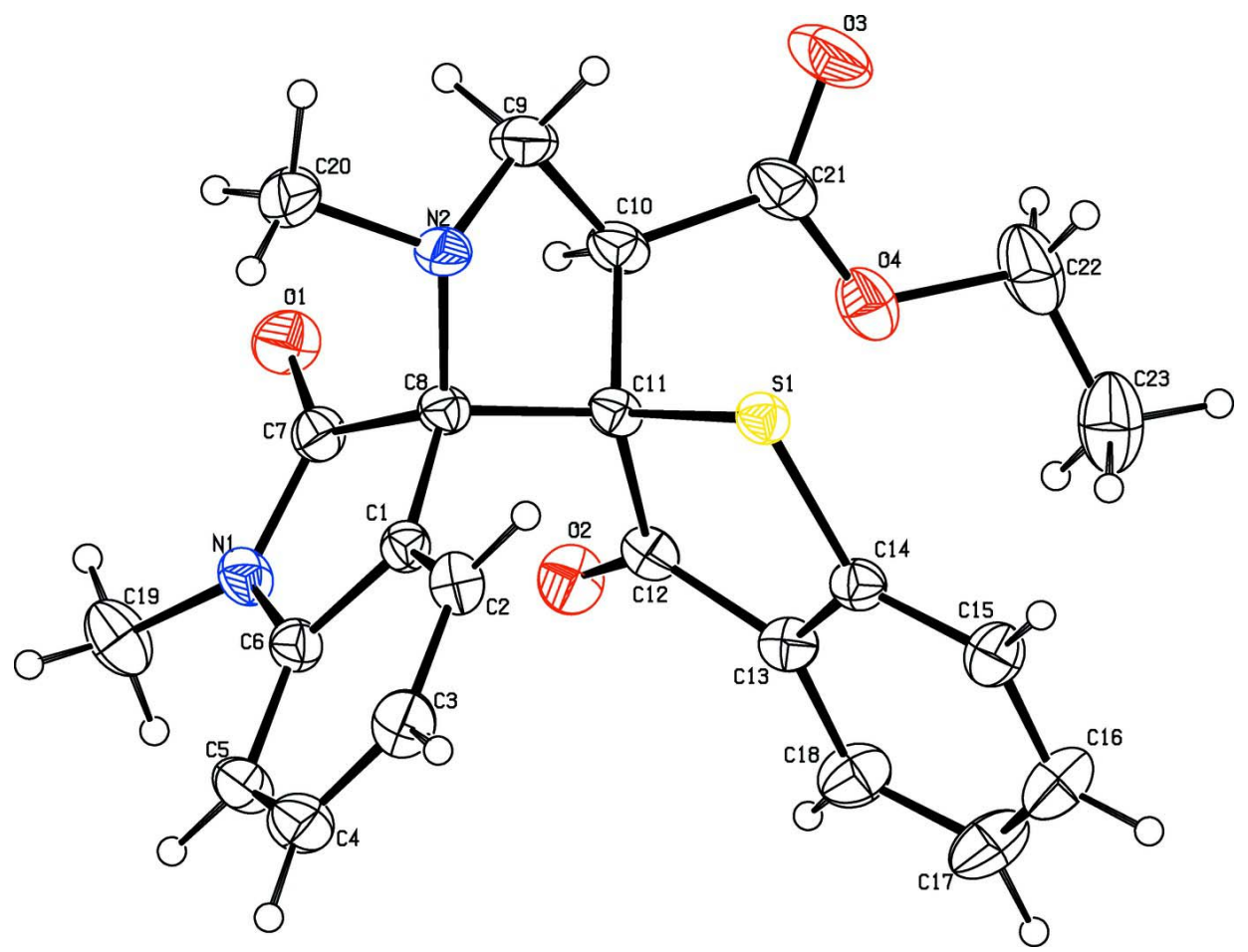

\section{Figure 1}

The molecular structure of the title compound with the atom numbering scheme. Displacement ellipsoids are drawn at the $30 \%$ probability level. 


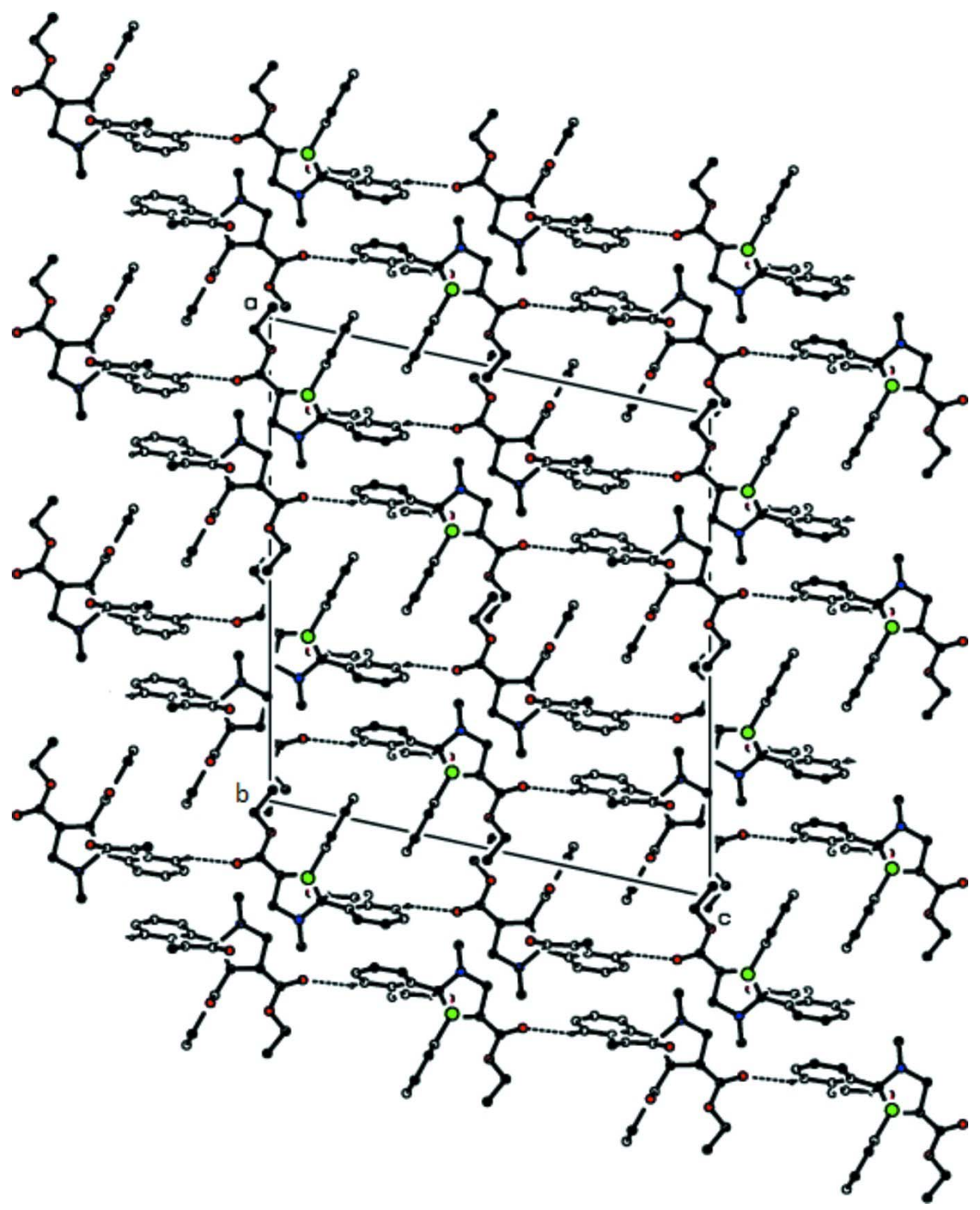

Figure 2

The crystal packing of the title compound viewed along the $b$ axis. Dashed lines shows the intermolecular $\mathrm{C}-\mathrm{H} \cdots \mathrm{O}$ hydrogen bonds. $\mathrm{H}$ atoms not involved in hydrogen bonding have been omitted for clarity.

Ethyl 1',1"-dimethyl-2",3-dioxo-3H-dispiro[benzo[b]thiophene-2,3'-pyrrolidine-2',3"'-indoline]-4'-carboxylate

Crystal data

$\mathrm{C}_{23} \mathrm{H}_{22} \mathrm{~N}_{2} \mathrm{O}_{4} \mathrm{~S}$

$M_{r}=422.49$

Monoclinic, $C 2 / c$

Hall symbol: $-\mathrm{C} 2 \mathrm{yc}$

$a=23.7049(11) \AA$

$b=8.2632(3) \AA$

$c=22.1003(8) \AA$

$\beta=102.337(2)^{\circ}$

$$
\begin{aligned}
& V=4229.0(3) \AA^{3} \\
& Z=8 \\
& F(000)=1776 \\
& D_{\mathrm{x}}=1.327 \mathrm{Mg} \mathrm{m}^{-3} \\
& \text { Mo } K \alpha \text { radiation, } \lambda=0.71073 \AA \\
& \text { Cell parameters from } 4634 \text { reflections } \\
& \theta=2.3-27.0^{\circ} \\
& \mu=0.19 \mathrm{~mm}^{-1}
\end{aligned}
$$


$T=293 \mathrm{~K}$

Block, colourless

Data collection

\section{Bruker Kappa APEXII CCD} diffractometer

Radiation source: fine-focus sealed tube

Graphite monochromator

$\omega$ and $\varphi$ scans

Absorption correction: multi-scan

(SADABS; Bruker, 2004)

$T_{\min }=0.896, T_{\max }=0.910$

Refinement

Refinement on $F^{2}$

Least-squares matrix: full

$R\left[F^{2}>2 \sigma\left(F^{2}\right)\right]=0.038$

$w R\left(F^{2}\right)=0.105$

$S=1.03$

4618 reflections

275 parameters

0 restraints

Primary atom site location: structure-invariant direct methods

Secondary atom site location: difference Fourier map

Special details

Geometry. All esds (except the esd in the dihedral angle between two 1.s. planes) are estimated using the full covariance matrix. The cell esds are taken into account individually in the estimation of esds in distances, angles and torsion angles; correlations between esds in cell parameters are only used when they are defined by crystal symmetry. An approximate (isotropic) treatment of cell esds is used for estimating esds involving l.s. planes.

Refinement. Refinement of $\mathrm{F}^{2}$ against ALL reflections. The weighted R-factor $\mathrm{wR}$ and goodness of fit $\mathrm{S}$ are based on $\mathrm{F}^{2}$, conventional R-factors $R$ are based on $F$, with $F$ set to zero for negative $F^{2}$. The threshold expression of $F^{2}>2 \operatorname{sigma}\left(F^{2}\right)$ is used only for calculating R-factors(gt) etc. and is not relevant to the choice of reflections for refinement. R-factors based on $\mathrm{F}^{2}$ are statistically about twice as large as those based on F, and R- factors based on ALL data will be even larger.

Fractional atomic coordinates and isotropic or equivalent isotropic displacement parameters $\left(\AA^{2}\right)$

\begin{tabular}{lllll}
\hline & $x$ & $y$ & $z$ & $U_{\text {iss }} * U_{\text {eq }}$ \\
\hline S1 & $0.141873(15)$ & $0.31779(4)$ & $0.413751(16)$ & $0.03357(12)$ \\
C11 & $0.13418(6)$ & $0.09712(17)$ & $0.41066(6)$ & $0.0316(3)$ \\
N1 & $0.16154(6)$ & $-0.18548(15)$ & $0.30761(6)$ & $0.0390(3)$ \\
C1 & $0.18787(6)$ & $0.08191(18)$ & $0.32013(6)$ & $0.0319(3)$ \\
O2 & $0.05531(5)$ & $-0.07835(14)$ & $0.36475(6)$ & $0.0499(3)$ \\
O1 & $0.17197(6)$ & $-0.27090(14)$ & $0.40812(6)$ & $0.0515(3)$ \\
C8 & $0.18360(6)$ & $0.02047(17)$ & $0.38341(6)$ & $0.0318(3)$ \\
C12 & $0.07265(6)$ & $0.05908(19)$ & $0.37426(7)$ & $0.0350(3)$ \\
C14 & $0.07062(6)$ & $0.34826(19)$ & $0.37340(7)$ & $0.0352(3)$ \\
C7 & $0.17122(6)$ & $-0.16405(18)$ & $0.37017(7)$ & $0.0364(3)$ \\
N2 & $0.23289(5)$ & $0.05295(16)$ & $0.43401(6)$ & $0.0384(3)$ \\
C13 & $0.03980(6)$ & $0.2070(2)$ & $0.35486(7)$ & $0.0380(3)$
\end{tabular}

22087 measured reflections

3869 reflections with $I>2 \sigma(I)$

$R_{\text {int }}=0.029$

$\theta_{\max }=27.0^{\circ}, \theta_{\min }=2.3^{\circ}$

$l=-22 \rightarrow 28$

Hydrogen site location: inferred from

neighbouring sites

$\mathrm{H}$-atom parameters constrained

where $P=\left(F_{0}^{2}+2 F_{\mathrm{c}}{ }^{2}\right) / 3$

$(\Delta / \sigma)_{\max }<0.001$

$\Delta \rho_{\max }=0.40$ e $\AA^{-3}$

$\Delta \rho_{\min }=-0.20$ e $\AA^{-3}$

Extinction correction: SHELXL97 (Sheldrick,

年, 2015$), \mathrm{Fc}^{*}=\mathrm{kFc}\left[1+0.001 \mathrm{xFc}^{2} \lambda^{3} / \sin (2 \theta)\right]^{-1 / 4}$

Extinction coefficient: 0.0025 (2)
4621 independent reflections 


\begin{tabular}{|c|c|c|c|c|}
\hline C6 & $0.17075(6)$ & $-0.04172(19)$ & $0.27721(7)$ & $0.0356(3)$ \\
\hline $\mathrm{C} 2$ & $0.20526(7)$ & $0.2287(2)$ & $0.30060(8)$ & 0.0395 \\
\hline $\mathrm{H} 2$ & 0.2187 & 0.3105 & 0.3290 & $0.047^{*}$ \\
\hline $\mathrm{O} 4$ & $0.06476(6)$ & $0.13849(16)$ & $0.49836(6)$ & 0.0556 \\
\hline $\mathrm{C} 10$ & $0.14909(7)$ & $0.0263(2)$ & $0.47706(7)$ & 0.0405 \\
\hline $\mathrm{H} 10$ & 0.1360 & -0.0865 & 0.4747 & $0.049 *$ \\
\hline C9 & $0.21520(8)$ & $0.0253(2)$ & $0.49324(8)$ & $0.0495(4)$ \\
\hline H9A & 0.2298 & 0.1104 & 0.5226 & $0.059^{*}$ \\
\hline H9B & 0.2296 & -0.0780 & 0.5110 & $0.059 *$ \\
\hline $\mathrm{C} 15$ & $0.04484(7)$ & 0.4995 (2) & $0.36049(8)$ & $0.0471(4)$ \\
\hline H15 & 0.0654 & 0.5942 & 0.3726 & $0.056^{*}$ \\
\hline $\mathrm{C} 20$ & $0.28698(7)$ & $-0.0239(2)$ & $0.42869(9)$ & $0.0541(5)$ \\
\hline $\mathrm{H} 20 \mathrm{~A}$ & 0.2826 & -0.1394 & 0.4290 & $0.081^{*}$ \\
\hline H20B & 0.3170 & 0.0083 & 0.4630 & $0.081 *$ \\
\hline $\mathrm{H} 20 \mathrm{C}$ & 0.2969 & 0.0088 & 0.3906 & $0.081 *$ \\
\hline $\mathrm{C} 18$ & $-0.01766(8)$ & $0.2148(2)$ & $0.32301(9)$ & $0.0539(5)$ \\
\hline H18 & -0.0384 & 0.1206 & 0.3105 & $0.065^{*}$ \\
\hline $\mathrm{C} 21$ & $0.12051(9)$ & $0.1125(2)$ & $0.52268(7)$ & 0.0498 \\
\hline $\mathrm{C} 5$ & $0.16713(7)$ & $-0.0205(2)$ & $0.21453(7)$ & $0.0474(4)$ \\
\hline H5 & 0.1547 & -0.1032 & 0.1863 & $0.057^{*}$ \\
\hline $\mathrm{C} 3$ & $0.20234(8)$ & $0.2522(2)$ & $0.23745(8)$ & 0.0489 (4) \\
\hline $\mathrm{H} 3$ & 0.2136 & 0.3508 & 0.2236 & $0.059^{*}$ \\
\hline $\mathrm{O} 3$ & $0.14426(8)$ & $0.1504(2)$ & $0.57445(6)$ & $0.0794(5)$ \\
\hline $\mathrm{C} 4$ & $0.18291(8)$ & $0.1304(3)$ & $0.19547(8)$ & $0.0530(5)$ \\
\hline $\mathrm{H} 4$ & 0.1803 & 0.1495 & 0.1535 & $0.064 *$ \\
\hline C19 & $0.15024(10)$ & $-0.3419(2)$ & $0.27783(10)$ & $0.0598(5)$ \\
\hline H19A & 0.1551 & -0.4252 & 0.3088 & $0.090^{*}$ \\
\hline H19B & 0.1767 & -0.3596 & 0.2511 & $0.090^{*}$ \\
\hline H19C & 0.1114 & -0.3445 & 0.2538 & $0.090^{*}$ \\
\hline $\mathrm{C} 16$ & $-0.01201(9)$ & $0.5045(3)$ & $0.32919(11)$ & $0.0646(5)$ \\
\hline H16 & -0.0300 & 0.6043 & 0.3204 & $0.078^{*}$ \\
\hline $\mathrm{C} 17$ & $-0.04313(8)$ & $0.3642(3)$ & $0.31035(11)$ & $0.0686(6)$ \\
\hline H17 & -0.0814 & 0.3713 & 0.2891 & $0.082 *$ \\
\hline $\mathrm{C} 22$ & $0.03086(12)$ & $0.2257(3)$ & $0.53552(11)$ & $0.0763(7)$ \\
\hline $\mathrm{H} 22 \mathrm{~A}$ & 0.0519 & 0.3201 & 0.5544 & $0.092 *$ \\
\hline $\mathrm{H} 22 \mathrm{~B}$ & 0.0230 & 0.1565 & 0.5682 & $0.092 *$ \\
\hline $\mathrm{C} 23$ & $-0.02388(11)$ & $0.2757(3)$ & $0.49378(13)$ & $0.0819(7)$ \\
\hline $\mathrm{H} 23 \mathrm{~A}$ & -0.0156 & 0.3458 & 0.4621 & $0.123 *$ \\
\hline $\mathrm{H} 23 \mathrm{~B}$ & -0.0475 & 0.3320 & 0.5172 & $0.123 *$ \\
\hline $\mathrm{H} 23 \mathrm{C}$ & -0.0440 & 0.1815 & 0.4749 & $0.123 *$ \\
\hline
\end{tabular}

Atomic displacement parameters $\left(\AA^{2}\right)$

\begin{tabular}{lllllll}
\hline & $U^{11}$ & $U^{22}$ & $U^{33}$ & $U^{12}$ & $U^{13}$ & $U^{23}$ \\
\hline S1 & $0.0366(2)$ & $0.0301(2)$ & $0.03283(19)$ & $-0.00394(14)$ & $0.00468(14)$ & $-0.00350(15)$ \\
C11 & $0.0373(7)$ & $0.0291(7)$ & $0.0280(6)$ & $-0.0048(6)$ & $0.0063(5)$ & $-0.0004(6)$ \\
N1 & $0.0449(7)$ & $0.0305(7)$ & $0.0405(7)$ & $-0.0051(5)$ & $0.0066(5)$ & $-0.0084(6)$ \\
C1 & $0.0295(7)$ & $0.0325(8)$ & $0.0336(7)$ & $0.0001(5)$ & $0.0066(5)$ & $-0.0022(6)$
\end{tabular}


supporting information

\begin{tabular}{lllllll}
$\mathrm{O} 2$ & $0.0461(6)$ & $0.0378(7)$ & $0.0646(8)$ & $-0.0129(5)$ & $0.0090(6)$ & $-0.0061(6)$ \\
$\mathrm{O} 1$ & $0.0686(8)$ & $0.0314(6)$ & $0.0514(7)$ & $-0.0028(5)$ & $0.0059(6)$ & $0.0072(6)$ \\
$\mathrm{C} 8$ & $0.0346(7)$ & $0.0279(7)$ & $0.0309(7)$ & $-0.0026(6)$ & $0.0028(5)$ & $-0.0017(6)$ \\
$\mathrm{C} 12$ & $0.0363(7)$ & $0.0380(8)$ & $0.0319(7)$ & $-0.0083(6)$ & $0.0103(6)$ & $-0.0018(6)$ \\
$\mathrm{C} 14$ & $0.0354(7)$ & $0.0394(8)$ & $0.0314(7)$ & $-0.0017(6)$ & $0.0087(6)$ & $0.0023(6)$ \\
$\mathrm{C} 7$ & $0.0374(8)$ & $0.0297(8)$ & $0.0402(8)$ & $-0.0012(6)$ & $0.0042(6)$ & $-0.0028(7)$ \\
$\mathrm{N} 2$ & $0.0368(7)$ & $0.0393(7)$ & $0.0344(6)$ & $-0.0001(5)$ & $-0.0027(5)$ & $-0.0052(6)$ \\
$\mathrm{C} 13$ & $0.0351(7)$ & $0.0424(9)$ & $0.0363(8)$ & $-0.0039(6)$ & $0.0074(6)$ & $0.0016(7)$ \\
$\mathrm{C} 6$ & $0.0324(7)$ & $0.0380(8)$ & $0.0357(7)$ & $-0.0004(6)$ & $0.0056(6)$ & $-0.0049(6)$ \\
$\mathrm{C} 2$ & $0.0405(8)$ & $0.0361(8)$ & $0.0447(9)$ & $-0.0032(6)$ & $0.0153(7)$ & $-0.0022(7)$ \\
O4 & $0.0676(8)$ & $0.0583(8)$ & $0.0484(7)$ & $-0.0018(6)$ & $0.0292(6)$ & $-0.0051(6)$ \\
C10 & $0.0561(9)$ & $0.0360(8)$ & $0.0286(7)$ & $-0.0033(7)$ & $0.0071(6)$ & $0.0036(6)$ \\
C9 & $0.0574(10)$ & $0.0516(10)$ & $0.0339(8)$ & $0.0029(8)$ & $-0.0028(7)$ & $0.0021(8)$ \\
C15 & $0.0486(9)$ & $0.0402(9)$ & $0.0527(10)$ & $0.0024(7)$ & $0.0116(8)$ & $0.0048(8)$ \\
C20 & $0.0412(9)$ & $0.0573(11)$ & $0.0574(11)$ & $0.0070(8)$ & $-0.0038(8)$ & $-0.0107(9)$ \\
C18 & $0.0388(9)$ & $0.0560(11)$ & $0.0624(11)$ & $-0.0086(8)$ & $0.0010(8)$ & $0.0020(9)$ \\
C21 & $0.0757(12)$ & $0.0444(10)$ & $0.0326(8)$ & $-0.0061(9)$ & $0.0187(8)$ & $0.0056(7)$ \\
C5 & $0.0516(9)$ & $0.0556(11)$ & $0.0341(8)$ & $0.0001(8)$ & $0.0075(7)$ & $-0.0095(8)$ \\
C3 & $0.0518(10)$ & $0.0489(10)$ & $0.0511(10)$ & $0.0011(8)$ & $0.0223(8)$ & $0.0104(8)$ \\
O3 & $0.1083(13)$ & $0.0999(13)$ & $0.0299(6)$ & $0.0017(10)$ & $0.0146(7)$ & $-0.0060(7)$ \\
C4 & $0.0585(11)$ & $0.0672(12)$ & $0.0359(8)$ & $0.0068(9)$ & $0.0160(7)$ & $0.0067(9)$ \\
C19 & $0.0756(13)$ & $0.0414(10)$ & $0.0633(12)$ & $-0.0176(9)$ & $0.0168(10)$ & $-0.0216(9)$ \\
C16 & $0.0524(11)$ & $0.0571(12)$ & $0.0803(14)$ & $0.0142(9)$ & $0.0051(10)$ & $0.0150(11)$ \\
C17 & $0.0396(9)$ & $0.0744(14)$ & $0.0835(15)$ & $0.0060(10)$ & $-0.0054(9)$ & $0.0138(12)$ \\
C22 & $0.1001(18)$ & $0.0769(16)$ & $0.0680(14)$ & $0.0025(13)$ & $0.0542(14)$ & $-0.0079(12)$ \\
C23 & $0.0883(17)$ & $0.0679(15)$ & $0.106(2)$ & $0.0066(13)$ & $0.0587(16)$ & $-0.0015(14)$ \\
& & & & & & \\
\hline & & & & & &
\end{tabular}

Geometric parameters $\left(\AA,{ }^{\circ}\right)$

\begin{tabular}{llll}
\hline $\mathrm{S} 1-\mathrm{C} 14$ & $1.7516(15)$ & $\mathrm{C} 10-\mathrm{H} 10$ & 0.9800 \\
$\mathrm{~S} 1-\mathrm{C} 11$ & $1.8324(15)$ & $\mathrm{C} 9-\mathrm{H} 9 \mathrm{~A}$ & 0.9700 \\
$\mathrm{C} 11-\mathrm{C} 12$ & $1.540(2)$ & $\mathrm{C} 9-\mathrm{H} 9 \mathrm{~B}$ & 0.9700 \\
$\mathrm{C} 11-\mathrm{C} 10$ & $1.5487(19)$ & $\mathrm{C} 15-\mathrm{C} 16$ & $1.378(3)$ \\
$\mathrm{C} 11-\mathrm{C} 8$ & $1.561(2)$ & $\mathrm{C} 15-\mathrm{H} 15$ & 0.9300 \\
$\mathrm{~N} 1-\mathrm{C} 7$ & $1.364(2)$ & $\mathrm{C} 20-\mathrm{H} 20 \mathrm{~A}$ & 0.9600 \\
$\mathrm{~N} 1-\mathrm{C} 6$ & $1.405(2)$ & $\mathrm{C} 20-\mathrm{H} 20 \mathrm{~B}$ & 0.9600 \\
$\mathrm{~N} 1-\mathrm{C} 19$ & $1.449(2)$ & $\mathrm{C} 20-\mathrm{H} 20 \mathrm{C}$ & 0.9600 \\
$\mathrm{C} 1-\mathrm{C} 2$ & $1.380(2)$ & $\mathrm{C} 18-\mathrm{C} 17$ & $1.377(3)$ \\
$\mathrm{C} 1-\mathrm{C} 6$ & $1.394(2)$ & $\mathrm{C} 18-\mathrm{H} 18$ & 0.9300 \\
$\mathrm{C} 1-\mathrm{C} 8$ & $1.511(2)$ & $\mathrm{C} 21-\mathrm{O} 3$ & $1.204(2)$ \\
$\mathrm{O} 2-\mathrm{C} 12$ & $1.2107(18)$ & $\mathrm{C} 5-\mathrm{C} 4$ & $1.393(3)$ \\
$\mathrm{O} 1-\mathrm{C} 7$ & $1.2153(19)$ & $\mathrm{C} 5-\mathrm{H} 5$ & 0.9300 \\
$\mathrm{C} 8-\mathrm{N} 2$ & $1.4592(18)$ & $\mathrm{C} 3-\mathrm{C} 4$ & $1.379(3)$ \\
$\mathrm{C} 8-\mathrm{C} 7$ & $1.568(2)$ & $\mathrm{C} 3-\mathrm{H} 3$ & 0.9300 \\
$\mathrm{C} 12-\mathrm{C} 13$ & $1.464(2)$ & $\mathrm{C} 4-\mathrm{H} 4$ & 0.9300 \\
$\mathrm{C} 14-\mathrm{C} 13$ & $1.391(2)$ & $\mathrm{C} 19-\mathrm{H} 19 \mathrm{~A}$ & 0.9600 \\
$\mathrm{C} 14-\mathrm{C} 15$ & $1.394(2)$ & $\mathrm{C} 19-\mathrm{H} 19 \mathrm{~B}$ & 0.9600 \\
$\mathrm{~N} 2-\mathrm{C} 20$ & $1.458(2)$ & $\mathrm{C} 19-\mathrm{H} 19 \mathrm{C}$ & 0.9600
\end{tabular}




\begin{tabular}{|c|c|c|c|}
\hline $\mathrm{N} 2-\mathrm{C} 9$ & $1.475(2)$ & $\mathrm{C} 16-\mathrm{C} 17$ & $1.389(3)$ \\
\hline $\mathrm{C} 13-\mathrm{C} 18$ & $1.395(2)$ & $\mathrm{C} 16-\mathrm{H} 16$ & 0.9300 \\
\hline $\mathrm{C} 6-\mathrm{C} 5$ & $1.381(2)$ & $\mathrm{C} 17-\mathrm{H} 17$ & 0.9300 \\
\hline $\mathrm{C} 2-\mathrm{C} 3$ & $1.396(2)$ & $\mathrm{C} 22-\mathrm{C} 23$ & $1.481(4)$ \\
\hline $\mathrm{C} 2-\mathrm{H} 2$ & 0.9300 & $\mathrm{C} 22-\mathrm{H} 22 \mathrm{~A}$ & 0.9700 \\
\hline $\mathrm{O} 4-\mathrm{C} 21$ & $1.333(2)$ & $\mathrm{C} 22-\mathrm{H} 22 \mathrm{~B}$ & 0.9700 \\
\hline $\mathrm{O} 4-\mathrm{C} 22$ & $1.457(2)$ & $\mathrm{C} 23-\mathrm{H} 23 \mathrm{~A}$ & 0.9600 \\
\hline $\mathrm{C} 10-\mathrm{C} 21$ & $1.508(2)$ & $\mathrm{C} 23-\mathrm{H} 23 \mathrm{~B}$ & 0.9600 \\
\hline $\mathrm{C} 10-\mathrm{C} 9$ & $1.531(2)$ & $\mathrm{C} 23-\mathrm{H} 23 \mathrm{C}$ & 0.9600 \\
\hline $\mathrm{C} 14-\mathrm{S} 1-\mathrm{C} 11$ & $92.67(7)$ & $\mathrm{C} 10-\mathrm{C} 9-\mathrm{H} 9 \mathrm{~B}$ & 110.7 \\
\hline $\mathrm{C} 12-\mathrm{C} 11-\mathrm{C} 10$ & $114.63(12)$ & $\mathrm{H} 9 \mathrm{~A}-\mathrm{C} 9-\mathrm{H} 9 \mathrm{~B}$ & 108.8 \\
\hline $\mathrm{C} 12-\mathrm{C} 11-\mathrm{C} 8$ & $114.87(11)$ & $\mathrm{C} 16-\mathrm{C} 15-\mathrm{C} 14$ & $117.94(17)$ \\
\hline $\mathrm{C} 10-\mathrm{C} 11-\mathrm{C} 8$ & $100.04(11)$ & $\mathrm{C} 16-\mathrm{C} 15-\mathrm{H} 15$ & 121.0 \\
\hline $\mathrm{C} 12-\mathrm{C} 11-\mathrm{S} 1$ & $107.34(10)$ & $\mathrm{C} 14-\mathrm{C} 15-\mathrm{H} 15$ & 121.0 \\
\hline $\mathrm{C} 10-\mathrm{C} 11-\mathrm{S} 1$ & $109.86(10)$ & $\mathrm{N} 2-\mathrm{C} 20-\mathrm{H} 20 \mathrm{~A}$ & 109.5 \\
\hline $\mathrm{C} 8-\mathrm{C} 11-\mathrm{S} 1$ & $109.95(9)$ & $\mathrm{N} 2-\mathrm{C} 20-\mathrm{H} 20 \mathrm{~B}$ & 109.5 \\
\hline $\mathrm{C} 7-\mathrm{N} 1-\mathrm{C} 6$ & $111.44(12)$ & $\mathrm{H} 20 \mathrm{~A}-\mathrm{C} 20-\mathrm{H} 20 \mathrm{~B}$ & 109.5 \\
\hline $\mathrm{C} 7-\mathrm{N} 1-\mathrm{C} 19$ & $123.43(14)$ & $\mathrm{N} 2-\mathrm{C} 20-\mathrm{H} 20 \mathrm{C}$ & 109.5 \\
\hline $\mathrm{C} 6-\mathrm{N} 1-\mathrm{C} 19$ & $124.72(14)$ & $\mathrm{H} 20 \mathrm{~A}-\mathrm{C} 20-\mathrm{H} 20 \mathrm{C}$ & 109.5 \\
\hline $\mathrm{C} 2-\mathrm{C} 1-\mathrm{C} 6$ & $119.63(14)$ & $\mathrm{H} 20 \mathrm{~B}-\mathrm{C} 20-\mathrm{H} 20 \mathrm{C}$ & 109.5 \\
\hline $\mathrm{C} 2-\mathrm{C} 1-\mathrm{C} 8$ & $131.94(13)$ & $\mathrm{C} 17-\mathrm{C} 18-\mathrm{C} 13$ & 118.84 \\
\hline $\mathrm{C} 6-\mathrm{C} 1-\mathrm{C} 8$ & $108.43(12)$ & $\mathrm{C} 17-\mathrm{C} 18-\mathrm{H} 18$ & 120.6 \\
\hline $\mathrm{N} 2-\mathrm{C} 8-\mathrm{C} 1$ & $116.51(12)$ & $\mathrm{C} 13-\mathrm{C} 18-\mathrm{H} 18$ & 120.6 \\
\hline $\mathrm{N} 2-\mathrm{C} 8-\mathrm{C} 11$ & $100.00(11)$ & $\mathrm{O} 3-\mathrm{C} 21-\mathrm{O} 4$ & $124.29(18)$ \\
\hline $\mathrm{C} 1-\mathrm{C} 8-\mathrm{C} 11$ & $115.14(11)$ & $\mathrm{O} 3-\mathrm{C} 21-\mathrm{C} 10$ & $124.94(19)$ \\
\hline $\mathrm{N} 2-\mathrm{C} 8-\mathrm{C} 7$ & $114.14(12)$ & $\mathrm{O} 4-\mathrm{C} 21-\mathrm{C} 10$ & $110.76(14)$ \\
\hline $\mathrm{C} 1-\mathrm{C} 8-\mathrm{C} 7$ & $101.59(11)$ & $\mathrm{C} 6-\mathrm{C} 5-\mathrm{C} 4$ & $117.01(16)$ \\
\hline $\mathrm{C} 11-\mathrm{C} 8-\mathrm{C} 7$ & $109.90(11)$ & $\mathrm{C} 6-\mathrm{C} 5-\mathrm{H} 5$ & 121.5 \\
\hline $\mathrm{O} 2-\mathrm{C} 12-\mathrm{C} 13$ & $126.37(14)$ & $\mathrm{C} 4-\mathrm{C} 5-\mathrm{H} 5$ & 121.5 \\
\hline $\mathrm{O} 2-\mathrm{C} 12-\mathrm{C} 11$ & $122.04(14)$ & $\mathrm{C} 4-\mathrm{C} 3-\mathrm{C} 2$ & $120.62(17)$ \\
\hline $\mathrm{C} 13-\mathrm{C} 12-\mathrm{C} 11$ & $111.58(12)$ & $\mathrm{C} 4-\mathrm{C} 3-\mathrm{H} 3$ & 119.7 \\
\hline $\mathrm{C} 13-\mathrm{C} 14-\mathrm{C} 15$ & $120.82(14)$ & $\mathrm{C} 2-\mathrm{C} 3-\mathrm{H} 3$ & 119.7 \\
\hline $\mathrm{C} 13-\mathrm{C} 14-\mathrm{S} 1$ & $114.70(12)$ & $\mathrm{C} 3-\mathrm{C} 4-\mathrm{C} 5$ & $121.49(16)$ \\
\hline $\mathrm{C} 15-\mathrm{C} 14-\mathrm{S} 1$ & $124.48(13)$ & $\mathrm{C} 3-\mathrm{C} 4-\mathrm{H} 4$ & 119.3 \\
\hline $\mathrm{O} 1-\mathrm{C} 7-\mathrm{N} 1$ & $125.43(14)$ & $\mathrm{C} 5-\mathrm{C} 4-\mathrm{H} 4$ & 119.3 \\
\hline $\mathrm{O} 1-\mathrm{C} 7-\mathrm{C} 8$ & $127.09(14)$ & $\mathrm{N} 1-\mathrm{C} 19-\mathrm{H} 19 \mathrm{~A}$ & 109.5 \\
\hline $\mathrm{N} 1-\mathrm{C} 7-\mathrm{C} 8$ & $107.43(12)$ & $\mathrm{N} 1-\mathrm{C} 19-\mathrm{H} 19 \mathrm{~B}$ & 109.5 \\
\hline $\mathrm{C} 20-\mathrm{N} 2-\mathrm{C} 8$ & $115.60(12)$ & $\mathrm{H} 19 \mathrm{~A}-\mathrm{C} 19-\mathrm{H} 19 \mathrm{~B}$ & 109.5 \\
\hline $\mathrm{C} 20-\mathrm{N} 2-\mathrm{C} 9$ & $115.19(13)$ & $\mathrm{N} 1-\mathrm{C} 19-\mathrm{H} 19 \mathrm{C}$ & 109.5 \\
\hline $\mathrm{C} 8-\mathrm{N} 2-\mathrm{C} 9$ & $108.58(12)$ & $\mathrm{H} 19 \mathrm{~A}-\mathrm{C} 19-\mathrm{H} 19 \mathrm{C}$ & 109.5 \\
\hline $\mathrm{C} 14-\mathrm{C} 13-\mathrm{C} 18$ & $120.33(15)$ & $\mathrm{H} 19 \mathrm{~B}-\mathrm{C} 19-\mathrm{H} 19 \mathrm{C}$ & 109.5 \\
\hline $\mathrm{C} 14-\mathrm{C} 13-\mathrm{C} 12$ & $113.66(13)$ & $\mathrm{C} 15-\mathrm{C} 16-\mathrm{C} 17$ & $121.70(18)$ \\
\hline $\mathrm{C} 18-\mathrm{C} 13-\mathrm{C} 12$ & $125.96(15)$ & $\mathrm{C} 15-\mathrm{C} 16-\mathrm{H} 16$ & 119.2 \\
\hline $\mathrm{C} 5-\mathrm{C} 6-\mathrm{C} 1$ & $122.43(15)$ & $\mathrm{C} 17-\mathrm{C} 16-\mathrm{H} 16$ & 119.2 \\
\hline $\mathrm{C} 5-\mathrm{C} 6-\mathrm{N} 1$ & $127.21(15)$ & $\mathrm{C} 18-\mathrm{C} 17-\mathrm{C} 16$ & $120.38(17)$ \\
\hline $\mathrm{C} 1-\mathrm{C} 6-\mathrm{N} 1$ & $110.28(13)$ & $\mathrm{C} 18-\mathrm{C} 17-\mathrm{H} 17$ & 119.8 \\
\hline $\mathrm{C} 1-\mathrm{C} 2-\mathrm{C} 3$ & $118.71(15)$ & $\mathrm{C} 16-\mathrm{C} 17-\mathrm{H} 17$ & 119.8 \\
\hline
\end{tabular}




\begin{tabular}{|c|c|c|c|}
\hline $\mathrm{C} 1-\mathrm{C} 2-\mathrm{H} 2$ & 120.6 & $\mathrm{O} 4-\mathrm{C} 22-\mathrm{C} 23$ & $107.59(18)$ \\
\hline $\mathrm{C} 3-\mathrm{C} 2-\mathrm{H} 2$ & 120.6 & $\mathrm{O} 4-\mathrm{C} 22-\mathrm{H} 22 \mathrm{~A}$ & 110.2 \\
\hline $\mathrm{C} 21-\mathrm{O} 4-\mathrm{C} 22$ & $118.09(16)$ & $\mathrm{C} 23-\mathrm{C} 22-\mathrm{H} 22 \mathrm{~A}$ & 110.2 \\
\hline $\mathrm{C} 21-\mathrm{C} 10-\mathrm{C} 9$ & $115.47(14)$ & $\mathrm{O} 4-\mathrm{C} 22-\mathrm{H} 22 \mathrm{~B}$ & 110.2 \\
\hline $\mathrm{C} 21-\mathrm{C} 10-\mathrm{C} 11$ & $114.26(13)$ & $\mathrm{C} 23-\mathrm{C} 22-\mathrm{H} 22 \mathrm{~B}$ & 110.2 \\
\hline $\mathrm{C} 9-\mathrm{C} 10-\mathrm{C} 11$ & $103.79(12)$ & $\mathrm{H} 22 \mathrm{~A}-\mathrm{C} 22-\mathrm{H} 22 \mathrm{~B}$ & 108.5 \\
\hline $\mathrm{C} 21-\mathrm{C} 10-\mathrm{H} 10$ & 107.6 & $\mathrm{C} 22-\mathrm{C} 23-\mathrm{H} 23 \mathrm{~A}$ & 109.5 \\
\hline $\mathrm{C} 9-\mathrm{C} 10-\mathrm{H} 10$ & 107.6 & $\mathrm{C} 22-\mathrm{C} 23-\mathrm{H} 23 \mathrm{~B}$ & 109.5 \\
\hline $\mathrm{C} 11-\mathrm{C} 10-\mathrm{H} 10$ & 107.6 & $\mathrm{H} 23 \mathrm{~A}-\mathrm{C} 23-\mathrm{H} 23 \mathrm{~B}$ & 109.5 \\
\hline $\mathrm{N} 2-\mathrm{C} 9-\mathrm{C} 10$ & $105.23(12)$ & $\mathrm{C} 22-\mathrm{C} 23-\mathrm{H} 23 \mathrm{C}$ & 109.5 \\
\hline $\mathrm{N} 2-\mathrm{C} 9-\mathrm{H} 9 \mathrm{~A}$ & 110.7 & $\mathrm{H} 23 \mathrm{~A}-\mathrm{C} 23-\mathrm{H} 23 \mathrm{C}$ & 109.5 \\
\hline $\mathrm{C} 10-\mathrm{C} 9-\mathrm{H} 9 \mathrm{~A}$ & 110.7 & $\mathrm{H} 23 \mathrm{~B}-\mathrm{C} 23-\mathrm{H} 23 \mathrm{C}$ & 109.5 \\
\hline $\mathrm{N} 2-\mathrm{C} 9-\mathrm{H} 9 \mathrm{~B}$ & 110.7 & & \\
\hline $\mathrm{C} 14-\mathrm{S} 1-\mathrm{C} 11-\mathrm{C} 12$ & $1.58(10)$ & $\mathrm{S} 1-\mathrm{C} 14-\mathrm{C} 13-\mathrm{C} 12$ & $1.56(17)$ \\
\hline $\mathrm{C} 14-\mathrm{S} 1-\mathrm{C} 11-\mathrm{C} 10$ & $-123.66(11)$ & $\mathrm{O} 2-\mathrm{C} 12-\mathrm{C} 13-\mathrm{C} 14$ & $178.78(15)$ \\
\hline $\mathrm{C} 14-\mathrm{S} 1-\mathrm{C} 11-\mathrm{C} 8$ & $127.17(10)$ & $\mathrm{C} 11-\mathrm{C} 12-\mathrm{C} 13-\mathrm{C} 14$ & $-0.26(18)$ \\
\hline $\mathrm{C} 2-\mathrm{C} 1-\mathrm{C} 8-\mathrm{N} 2$ & $-45.8(2)$ & $\mathrm{O} 2-\mathrm{C} 12-\mathrm{C} 13-\mathrm{C} 18$ & $1.3(3)$ \\
\hline $\mathrm{C} 6-\mathrm{C} 1-\mathrm{C} 8-\mathrm{N} 2$ & $133.48(13)$ & $\mathrm{C} 11-\mathrm{C} 12-\mathrm{C} 13-\mathrm{C} 18$ & $-177.73(15)$ \\
\hline $\mathrm{C} 2-\mathrm{C} 1-\mathrm{C} 8-\mathrm{C} 11$ & $70.9(2)$ & $\mathrm{C} 2-\mathrm{C} 1-\mathrm{C} 6-\mathrm{C} 5$ & $-3.9(2)$ \\
\hline $\mathrm{C} 6-\mathrm{C} 1-\mathrm{C} 8-\mathrm{C} 11$ & $-109.88(13)$ & $\mathrm{C} 8-\mathrm{C} 1-\mathrm{C} 6-\mathrm{C} 5$ & $176.69(14)$ \\
\hline $\mathrm{C} 2-\mathrm{C} 1-\mathrm{C} 8-\mathrm{C} 7$ & $-170.46(15)$ & $\mathrm{C} 2-\mathrm{C} 1-\mathrm{C} 6-\mathrm{N} 1$ & $172.93(13)$ \\
\hline $\mathrm{C} 6-\mathrm{C} 1-\mathrm{C} 8-\mathrm{C} 7$ & $8.80(14)$ & $\mathrm{C} 8-\mathrm{C} 1-\mathrm{C} 6-\mathrm{N} 1$ & $-6.44(16)$ \\
\hline $\mathrm{C} 12-\mathrm{C} 11-\mathrm{C} 8-\mathrm{N} 2$ & $-169.56(12)$ & $\mathrm{C} 7-\mathrm{N} 1-\mathrm{C} 6-\mathrm{C} 5$ & $177.34(15)$ \\
\hline $\mathrm{C} 10-\mathrm{C} 11-\mathrm{C} 8-\mathrm{N} 2$ & $-46.29(13)$ & $\mathrm{C} 19-\mathrm{N} 1-\mathrm{C} 6-\mathrm{C} 5$ & $4.5(3)$ \\
\hline $\mathrm{S} 1-\mathrm{C} 11-\mathrm{C} 8-\mathrm{N} 2$ & $69.26(11)$ & $\mathrm{C} 7-\mathrm{N} 1-\mathrm{C} 6-\mathrm{C} 1$ & $0.65(17)$ \\
\hline $\mathrm{C} 12-\mathrm{C} 11-\mathrm{C} 8-\mathrm{C} 1$ & $64.75(16)$ & $\mathrm{C} 19-\mathrm{N} 1-\mathrm{C} 6-\mathrm{C} 1$ & $-172.18(15)$ \\
\hline $\mathrm{C} 10-\mathrm{C} 11-\mathrm{C} 8-\mathrm{C} 1$ & $-171.98(12)$ & $\mathrm{C} 6-\mathrm{C} 1-\mathrm{C} 2-\mathrm{C} 3$ & $3.3(2)$ \\
\hline $\mathrm{S} 1-\mathrm{C} 11-\mathrm{C} 8-\mathrm{C} 1$ & $-56.43(14)$ & $\mathrm{C} 8-\mathrm{C} 1-\mathrm{C} 2-\mathrm{C} 3$ & $-177.51(15)$ \\
\hline $\mathrm{C} 12-\mathrm{C} 11-\mathrm{C} 8-\mathrm{C} 7$ & $-49.19(16)$ & $\mathrm{C} 12-\mathrm{C} 11-\mathrm{C} 10-\mathrm{C} 21$ & $-73.44(17)$ \\
\hline $\mathrm{C} 10-\mathrm{C} 11-\mathrm{C} 8-\mathrm{C} 7$ & $74.08(13)$ & $\mathrm{C} 8-\mathrm{C} 11-\mathrm{C} 10-\mathrm{C} 21$ & $163.12(13)$ \\
\hline $\mathrm{S} 1-\mathrm{C} 11-\mathrm{C} 8-\mathrm{C} 7$ & $-170.37(9)$ & $\mathrm{S} 1-\mathrm{C} 11-\mathrm{C} 10-\mathrm{C} 21$ & $47.50(16)$ \\
\hline $\mathrm{C} 10-\mathrm{C} 11-\mathrm{C} 12-\mathrm{O} 2$ & $-57.80(19)$ & $\mathrm{C} 12-\mathrm{C} 11-\mathrm{C} 10-\mathrm{C} 9$ & $159.94(13)$ \\
\hline $\mathrm{C} 8-\mathrm{C} 11-\mathrm{C} 12-\mathrm{O} 2$ & $57.28(19)$ & $\mathrm{C} 8-\mathrm{C} 11-\mathrm{C} 10-\mathrm{C} 9$ & $36.51(14)$ \\
\hline $\mathrm{S} 1-\mathrm{C} 11-\mathrm{C} 12-\mathrm{O} 2$ & $179.88(12)$ & $\mathrm{S} 1-\mathrm{C} 11-\mathrm{C} 10-\mathrm{C} 9$ & $-79.11(13)$ \\
\hline $\mathrm{C} 10-\mathrm{C} 11-\mathrm{C} 12-\mathrm{C} 13$ & $121.29(14)$ & $\mathrm{C} 20-\mathrm{N} 2-\mathrm{C} 9-\mathrm{C} 10$ & $-148.67(14)$ \\
\hline $\mathrm{C} 8-\mathrm{C} 11-\mathrm{C} 12-\mathrm{C} 13$ & $-123.63(13)$ & $\mathrm{C} 8-\mathrm{N} 2-\mathrm{C} 9-\mathrm{C} 10$ & $-17.23(17)$ \\
\hline $\mathrm{S} 1-\mathrm{C} 11-\mathrm{C} 12-\mathrm{C} 13$ & $-1.03(14)$ & $\mathrm{C} 21-\mathrm{C} 10-\mathrm{C} 9-\mathrm{N} 2$ & $-139.30(14)$ \\
\hline $\mathrm{C} 11-\mathrm{S} 1-\mathrm{C} 14-\mathrm{C} 13$ & $-1.87(12)$ & $\mathrm{C} 11-\mathrm{C} 10-\mathrm{C} 9-\mathrm{N} 2$ & $-13.45(17)$ \\
\hline $\mathrm{C} 11-\mathrm{S} 1-\mathrm{C} 14-\mathrm{C} 15$ & $177.39(14)$ & $\mathrm{C} 13-\mathrm{C} 14-\mathrm{C} 15-\mathrm{C} 16$ & $0.3(2)$ \\
\hline $\mathrm{C} 6-\mathrm{N} 1-\mathrm{C} 7-\mathrm{O} 1$ & $-172.45(15)$ & $\mathrm{S} 1-\mathrm{C} 14-\mathrm{C} 15-\mathrm{C} 16$ & $-178.89(14)$ \\
\hline $\mathrm{C} 19-\mathrm{N} 1-\mathrm{C} 7-\mathrm{O} 1$ & $0.5(3)$ & $\mathrm{C} 14-\mathrm{C} 13-\mathrm{C} 18-\mathrm{C} 17$ & $0.0(3)$ \\
\hline $\mathrm{C} 6-\mathrm{N} 1-\mathrm{C} 7-\mathrm{C} 8$ & $5.18(17)$ & $\mathrm{C} 12-\mathrm{C} 13-\mathrm{C} 18-\mathrm{C} 17$ & $177.33(18)$ \\
\hline $\mathrm{C} 19-\mathrm{N} 1-\mathrm{C} 7-\mathrm{C} 8$ & $178.12(15)$ & $\mathrm{C} 22-\mathrm{O} 4-\mathrm{C} 21-\mathrm{O} 3$ & $3.3(3)$ \\
\hline $\mathrm{N} 2-\mathrm{C} 8-\mathrm{C} 7-\mathrm{O} 1$ & $42.9(2)$ & $\mathrm{C} 22-\mathrm{O} 4-\mathrm{C} 21-\mathrm{C} 10$ & $-177.97(16)$ \\
\hline $\mathrm{C} 1-\mathrm{C} 8-\mathrm{C} 7-\mathrm{O} 1$ & $169.17(15)$ & $\mathrm{C} 9-\mathrm{C} 10-\mathrm{C} 21-\mathrm{O} 3$ & $-15.2(3)$ \\
\hline $\mathrm{C} 11-\mathrm{C} 8-\mathrm{C} 7-\mathrm{O} 1$ & $-68.47(19)$ & $\mathrm{C} 11-\mathrm{C} 10-\mathrm{C} 21-\mathrm{O} 3$ & $-135.47(19)$ \\
\hline $\mathrm{N} 2-\mathrm{C} 8-\mathrm{C} 7-\mathrm{N} 1$ & $-134.66(13)$ & $\mathrm{C} 9-\mathrm{C} 10-\mathrm{C} 21-\mathrm{O} 4$ & $166.10(14)$ \\
\hline
\end{tabular}




$\begin{array}{llll}\mathrm{C} 1-\mathrm{C} 8-\mathrm{C} 7-\mathrm{N} 1 & -8.42(15) & \mathrm{C} 11-\mathrm{C} 10-\mathrm{C} 21-\mathrm{O} 4 & 45.81(19) \\ \mathrm{C} 11-\mathrm{C} 8-\mathrm{C} 7-\mathrm{N} 1 & 113.95(13) & \mathrm{C} 1-\mathrm{C} 6-\mathrm{C} 5-\mathrm{C} 4 & 1.6(2) \\ \mathrm{C} 1-\mathrm{C} 8-\mathrm{N} 2-\mathrm{C} 20 & -63.90(18) & \mathrm{N} 1-\mathrm{C} 6-\mathrm{C} 5-\mathrm{C} 4 & -174.69(15) \\ \mathrm{C} 11-\mathrm{C} 8-\mathrm{N} 2-\mathrm{C} 20 & 171.35(14) & \mathrm{C} 1-\mathrm{C} 2-\mathrm{C} 3-\mathrm{C} 4 & -0.5(2) \\ \mathrm{C} 7-\mathrm{C} 8-\mathrm{N} 2-\mathrm{C} 20 & 54.10(18) & \mathrm{C} 2-\mathrm{C} 3-\mathrm{C} 4-\mathrm{C} 5 & -1.8(3) \\ \mathrm{C} 1-\mathrm{C} 8-\mathrm{N} 2-\mathrm{C} 9 & 164.88(13) & \mathrm{C} 6-\mathrm{C} 5-\mathrm{C} 4-\mathrm{C} 3 & 1.2(3) \\ \mathrm{C} 11-\mathrm{C} 8-\mathrm{N} 2-\mathrm{C} 9 & 40.13(14) & \mathrm{C} 14-\mathrm{C} 15-\mathrm{C} 16-\mathrm{C} 17 & -0.5(3) \\ \mathrm{C} 7-\mathrm{C} 8-\mathrm{N} 2-\mathrm{C} 9 & -77.11(16) & \mathrm{C} 13-\mathrm{C} 18-\mathrm{C} 17-\mathrm{C} 16 & -0.2(3) \\ \mathrm{C} 15-\mathrm{C} 14-\mathrm{C} 13-\mathrm{C} 18 & -0.1(2) & \mathrm{C} 15-\mathrm{C} 16-\mathrm{C} 17-\mathrm{C} 18 & 0.4(4) \\ \mathrm{S} 1-\mathrm{C} 14-\mathrm{C} 13-\mathrm{C} 18 & 179.19(13) & \mathrm{C} 21-\mathrm{O} 4-\mathrm{C} 22-\mathrm{C} 23 & 165.63(18) \\ \mathrm{C} 15-\mathrm{C} 14-\mathrm{C} 13-\mathrm{C} 12 & -177.72(14) & & \end{array}$

Hydrogen-bond geometry $\left(A,{ }^{\circ}\right)$

\begin{tabular}{lllll}
\hline$D-\mathrm{H}^{\cdots} \cdots A$ & $D-\mathrm{H}$ & $\mathrm{H} \cdots A$ & $D \cdots A$ & $D-\mathrm{H} \cdots A$ \\
\hline $\mathrm{C} 5-\mathrm{H} 5 \cdots \mathrm{O}^{\mathrm{i}}$ & 0.93 & 2.46 & $3.212(2)$ & 138 \\
\hline
\end{tabular}

Symmetry code: (i) $x,-y, z-1 / 2$. 